\title{
Severe acute respiratory infection caused by swine influenza virus in a child necessitating extracorporeal membrane oxygenation (ECMO), the Netherlands, October 2016
}

PLA Fraaij ${ }^{12}$, ED Wildschut ${ }^{3}$, RJ Houmes ${ }^{3}, \mathrm{CM} \mathrm{Swaan}^{4}$, CJ Hoebe $5^{6}$, HCC de Jonge $^{7}, \mathrm{P}$ Tolsma ${ }^{8}$, I de Kleer ${ }^{9}$, SD Pas $^{1}$, BB Oude Munnink ${ }^{1}$, MVT Phan ${ }^{1}$, TM Bestebroer ${ }^{1}$, RS Roosenhoff ${ }^{1}$, JJA van Kampen ${ }^{1}$, M Cotten ${ }^{1}$, N Beerens ${ }^{10}$, RAM Fouchier ${ }^{1}$, JH van den Kerkhof ${ }^{4}$, A Timen ${ }^{4}$, MP Koopmans ${ }^{1}$

1. Department of Viroscience Erasmus MC, Rotterdam, The Netherlands

2. Department of Pediatrics, Subdivision Infectious diseases and Immunology, Erasmus MC - Sophia, Rotterdam, The Netherlands

3. Intensive Care and Department of Pediatric Surgery, Erasmus MC-Sophia, Rotterdam, The Netherlands

4. Centre for Infectious Disease Control-National Institute for Public Health and the Environment, Bilthoven, The Netherlands

5. Department of Sexual Health, Infectious Diseases and Environmental Health, Public Health Service South Limburg, Geleen, The Netherlands

6. Faculty of Health, Medicine and Life Sciences Department of Medical Microbiology, Maastricht Infection Center (MINC), School of Public Health and Primary Care (CAPHRI), Maastricht University Medical Center (MUMC+), Maastricht, The Netherlands

7. Gemeentelijke Gezondheidsdienst Rotterdam-Rijnmond, Rotterdam, The Netherlands

8. Gemeentelijke Gezondheidsdienst Brabant zuidoost, Eindhoven, The Netherlands

9. Department of Paediatrics, Subdivision of pulmonary medicine, Erasmus MC - Sophia, Rotterdam, The Netherlands

10. Wageningen Bioveterinary reseach- Wageningen University and Research, Lelystad, the Netherlands

11. Wageningen University and Research, Wageningen the Netherlands

Correspondence: Pieter L. Fraaij (p.fraaij@erasmusmc.nl)

Citation style for this article:

Fraaij PLA, Wildschut ED, Houmes RJ, Swaan CM, Hoebe CJ, de Jonge HCC, Tolsma P, de Kleer I, Pas SD, Oude Munnink BB, Phan MVT, Bestebroer TM, Roosenhoff RS, van Kampen JJA, Cotten M, Beerens N, Fouchier RAM, van den Kerkhof JH, Timen A, Koopmans MP. Severe acute respiratory infection caused by swine influenza virus in a child necessitating extracorporeal membrane oxygenation (ECMO), the Netherlands, October 2016. Euro Surveill. 2016;21(48):pii=30416. DOI: http:// dx.doi.org/10.2807/1560-7917.ES.2016.21.48.30416

Article submitted on 14 November 2016 / accepted on 30 November 2016 / published on 01 December 2016

In October 2016, a severe infection with swine influenza $A\left(\mathrm{H}_{1} \mathrm{~N}_{1}\right)$ virus of the Eurasian avian lineage occurred in a child with a previous history of eczema in the Netherlands, following contact to pigs. The patient's condition deteriorated rapidly and required life support through extracorporeal membrane oxygenation. After start of oseltamivir treatment and removal of mucus plugs, the patient fully recovered. Monitoring of more than 80 close unprotected contacts revealed no secondary cases.

We here report a patient with severe acute respiratory infection as a result of swine influenza virus (SIV) infection in the Netherlands.

\section{Case description}

A school-aged patient with a previous history of mild eczema developed a respiratory tract infection in October 2016, a couple of days after visiting a pig farm. The child had entered the pigsty but had not been in direct contact with pigs. Despite early prescription of antibiotics by the general practitioner the child's clinical situation rapidly deteriorated. Within three days after onset of disease the child was transferred to a paediatric intensive care unit (PICU) for non-invasive ventilation support and intensive monitoring. Despite these efforts, the patient deteriorated further and was intubated in order to start mechanical ventilation. Bronchoscopy following intubation revealed large amounts of highly viscous mucus in the airways. Efforts to remove this mucus failed to improve ventilation. Mechanical ventilation became increasingly complex and it was decided to initiate veno-venous extracorporeal membrane oxygenation (ECMO) and to transfer to a quaternary PICU. Due to ECMO, blood oxygenation was secured and extensive bronchoscopy could be performed, during which topical DNAse (Dornase alpha, Pulmozyme, Roche) was instilled to decrease viscosity and facilitate removal of obstructing mucus plugs. On the following day, bronchoscopy was repeated and additional mucus was removed.

In the days following these procedures, the patient improved rapidly. ECMO was discontinued five days after start and the patient could be extubated. For the entire duration of hospitalisation, the patient had received broad-spectrum antibiotics, although all bacterial cultures remained negative. Throat swabs had been collected at initial admission and tested positive for influenza A virus, of which the quaternary PICU 
was informed on the day after the patient transfer. Oseltamivir treatment (6o mg twice daily) was started hours after initiation of ECMO and transport. It was continued for a total of 7 days when a nasal swab tested negative for influenza virus. At the time of submission of this report, the child was recovering well.

\section{Virological results}

The initial diagnostic routine was limited to testing for the influenza A virus matrix gene, without subtyping. In view of the severe course of illness, the child was resampled for repeated testing including typing of the haemagglutinin (HA) gene by quantitative real-time PCR for $\mathrm{H}_{1}$ (seasonal and pdm2009), $\mathrm{H}_{3}, \mathrm{H}_{5}, \mathrm{H}_{7}$ and Hy. All typing PCRs were negative.

We determined the full virus genome sequences of a cell culture isolate derived from a respiratory tract sample using Illumina MiSeq. All gene segments (GenBank accession numbers KY250316-KY250323) were $97-98 \%$ and $98-100 \%$ identical at, respectively, nucleotide and amino acid level to publicly available SIV sequences from the Netherlands (GISAID accession numbers EPI639351, EPI639914, EPI639917, EPI639930, EPI640657, EPI640912, EPI641210, EPI641215). The gene segments were all of the Eurasian avian $A\left(\mathrm{H}_{1} \mathrm{~N}_{1}\right)$ SIV lineage that has been circulating in pigs since 1979 [1]. Pigs at the farm visited by the patient tested positive for the same SIV (curation of full genome sequence data is in progress). The virus isolate from the patient, A/Netherlands/3315/2016, was sensitive to oseltamivir and zanamivir by NA-star neuraminidase inhibitor resistance detection assay (Applied Biosystems, Nieuwerkerk aan den IJssel, The Netherlands).

\section{Public health measures}

Zoonotic influenza is a notifiable disease in the Netherlands. Following confirmation of the zoonotic SIV infection, the national and relevant municipal public health authorities were notified and a teleconference was organised to decide on measures. The risk for human-to-human transmission was considered very low, given the enzootic presence of swine influenza viruses and the fact that zoonotic infections are seldom diagnosed.

In order to detect human-to-human transmission at an early stage, it was decided to contact all individuals that had been in close direct contact with the patient without wearing personal protective equipment, and monitor them for symptoms of possible SIV infection (cough, fever or conjunctivitis) for 10 days after exposure. In total, more than 80 contacts were monitored. These included the patient's family members living in the same household, persons living and working on the pig farm, and healthcare workers who cared for the patient without wearing personal protective equipment (i.e. before the influenza diagnosis). Six contacts developed mild respiratory symptoms including cough, coryza and conjunctivitis during the monitoring period but all tested negative for influenza A virus.
According to the international health regulations, this case has been notified to the European Union Member States and the European Centre for Disease Prevention and Control (ECDC) through the Early Warning and Response System (EWRS) and to the World Health Organization (WHO).

\section{Discussion}

Incidental cases of human infection with SIV have been reported worldwide since the late 1950s. Most of these were in individuals exposed to pigs. Apart from one isolated incident in military barracks in the United States (US), sustained and efficient human- to- human transmission had not been documented before 2009, when an influenza virus of swine origin triggered the first influenza pandemic of the 21st century. Indeed it is speculated that pigs may serve as a mixing vessel for the development of a pandemic influenza strain [2-5]. In addition, SIV infections account for roughly one third of all laboratory-confirmed zoonotic influenza events reported in the scientific literature [3]. This may be a gross underestimation of the actual number as there are no typical signs and symptoms that distinguish SIV infections in humans from those caused by seasonal influenza viruses [4]. Indeed several sero-epidemiological studies suggest that SIV infection in people with occupational swine exposure is common [6-8]. In the US, there is a routine surveillance for swine influenza in pigs, and 400 patients with a swine influenza infection have been reported through this system since 2005 [9]. Our case shows that careful assessment of airway disease in individuals exposed to pigs continues to be important, especially considering the importance of starting of antiviral treatment early.

\section{Conclusion}

We here describe that transmission of SIV to humans, though rare, can occur and cause severe disease requiring life support through ECMO. Monitoring of people in direct contact and not wearing personal protective equipment revealed no secondary cases.

\section{Acknowledgments}

We thank the parents for careful reading of and consent to publish the case description. PF and MK receive funding from the EU FP7 project PREPARE (\#602525). MK and RF receive funding from the EU FP7 project COMPARE(\#643476) and NIAID contract HHSN272201400008C. We thank the Global Initiative for Sharing All Influenza Data (GISAID) and all laboratories providing GISAID with sequence information on influenza $A$ viruses used in this analysis. We thank the Netherlands Food and Consumer Product Safety Authority (NVWA for their efforts.

\section{Conflict of interest}

None declared.

Authors' contributions 
All authors contributed to the text of the manuscript. PLF, EDW, RJH, IK took care of the patient. CJH, HCJ, PT were involved in case finding. PLF, SDP, TMB, RR, JJK, RAF, MVTP, BOM, MC, NB, MPK were involved in lab testing. HK, AT, CMS, were involved in outbreak management.

\section{References}

1. Watson SJ, Langat P, Reid SM, Lam TT, Cotten M, Kelly M, et al. Molecular Epidemiology and Evolution of Influenza Viruses Circulating within European Swine between 2009 and 2013. J Virol. 2015;89(19):9920-31. DOI: 10.1128/JVI.00840-15 PMID: 26202246

2. Top FH, Russell PK. Swine influenza A at Fort Dix, New Jersey (January-February 1976). IV. Summary and speculation.J Infect Dis. 1977;136(Suppl):S376-80. DOI: 10.1093/infdis/136. Supplement_3.S376 PMID: 342615

3. Freidl GS, Meijer A, de Bruin E, de Nardi M, Munoz O, Capua I, et al. Influenza at the animal-human interface: a review of the literature for virological evidence of human infection with swine or avian influenza viruses other than $\mathrm{A}\left(\mathrm{H}_{5} \mathrm{~N}_{1}\right)$ Euro Surveill. 2014;19(18):20793. DOI: 10.2807/1560-7917. ES2014.19.18.20793 PMID: 24832117

4. Myers KP, Olsen CW, Gray GC. Cases of swine influenza in humans: a review of the literature.Clin Infect Dis. 2007;44(8):1084-8. DOI: 10.1086/512813 PMID: 17366454

5. Rimmelzwaan GF, de Jong JC, Bestebroer TM, van Loon AM, Claas EC, Fouchier RA, et al. Antigenic and genetic characterization of swine influenza $A\left(\mathrm{H}_{1} \mathrm{~N}_{1}\right)$ viruses isolated from pneumonia patients in The Netherlands. Virology. 2001;282(2):301-6. DOI: 10.1006/viro.2000.0810 PMID: 11289812

6. Myers KP, Olsen CW, Setterquist SF, Capuano AW, Donham KJ, Thacker EL, et al. Are swine workers in the United States at increased risk of infection with zoonotic influenza virus? Clin Infect Dis. 2006;42(1):14-20. DOI: 10.1086/498977 PMID: 16323086

7. Olsen CW, Brammer L, Easterday BC, Arden N, Belay E, Baker I, et al. Serologic evidence of $\mathrm{H}_{1}$ swine Influenza virus infection in swine farm residents and employees. Emerg Infect Dis. 2002;8(8):814-9. DOI: 10.3201/eido808.010474 PMID: 12141967

8. Terebuh P, Olsen CW, Wright J, Klimov A, Karasin A, Todd $\mathrm{K}$, et al. Transmission of influenza A viruses between pigs and people, lowa, 2002-2004. Influenza Other Respi Viruses. 2010;4(6):387-96. DOI: 10.1111/j.1750-2659.2010.00175.x PMID: 20958933

9. Centers for Disease Control and Prevention (CDC). Reported infections with variant influenza viruses in the United States since 2005. Atlanta: CDC; 28 Nov 2016. Available from: http:// www.cdc.gov/flu/swineflu/variant-cases-us.htm

\section{License and copyright}

This is an open-access article distributed under the terms of the Creative Commons Attribution (CC BY 4.0) Licence. You may share and adapt the material, but must give appropriate credit to the source, provide a link to the licence, and indicate if changes were made.

This article is copyright of the authors, 2016. 